\title{
Practical Notes on the Measurement and Interpretation of Thermally Stimulated Discharge Currents in Biaxially Oriented Polypropylene
}

\author{
I. Rytöluoto ${ }^{1,2}$, M. Niittymäki ${ }^{2} \&$ K. Lahti ${ }^{2}$ \\ ${ }^{1}$ VTT Technical Research Centre of Finland (VTT), Tampere, Finland \\ ${ }^{2}$ Tampere University (TAU), Electrical Engineering, Tampere, Finland \\ ilkka.rytoluoto@tuni.fi
}

\begin{abstract}
The measurement and interpretation of high-field thermally stimulated depolarization current (TSDC) of capacitor-grade biaxially oriented polypropylene (BOPP) films are investigated in order to facilitate the derivation of trap parameters. Two main relaxations associable with the glass transition and detrapping of space charge from deep traps are observed, and their dependence on the polarization/depolarization conditions are studied. The complex bipolar nature of the space charge relaxation makes the derivation of trap parameters challenging. Lastly, a brief attempt is made to correlate the TSDC features with morphology and other short-term dielectric properties.
\end{abstract}

\section{Introduction}

TSDC measurement is a useful technique for studying charge storage and decay processes in polymer thin films. Generally, the TSDC technique involves $(i)$ high-field excitation of the polymeric specimen at elevated temperature, (ii) rapid cooling of the specimen to "freeze" the induced polarization/space charge and (iii) controlled discharge of the short-circuited specimen under linear heating condition. In principle, TSDC data allow the extraction of trapping parameters such as trap depth and density, making TSDC an attractive technique for exploring the inter-relationships between electronic structure (i.e. density of states) and bulk dielectric properties. Trap parameters determined by TSDC could, for instance, be incorporated into the bipolar charge transport model to facilitate understanding on high-field conductivity behavior of polymer nanocomposite films.

In practice however, a number of issues related to e.g. sample preparation, morphology, surface roughness, electrode-polymer interface as well as the sole analysis of the TSDC data itself make the measurement and interpretation of high-field TSDC of thin polymer films far from trivial. This paper overviews some of the aforementioned aspects based on recent TSDC studies carried out at TAU for various commercial capacitor-grade polypropylene thin films. Experimental complications related to the preparation and measurement of thin films, and some methods to overcome them, are presented. Moreover, various data analysis methods for extracting the charge trapping parameters from TSDC spectra (and the issues related to them) are reviewed, ranging from simple methods assuming single discrete relaxations to full-scale deconvolution of (quasi-) continuous trap bands by multi-peak fitting or other techniques.
Lastly, a brief attempt is made to correlate the TSDC features of various capacitor BOPP films (manufactured by both bubble and tenter processes) with their morphology and other short-term dielectric properties.

\section{Experimental}

\subsection{Materials and electrode deposition}

The studied materials, enlisted in Table 1, included various commercial BOPP films intended for metallized film and oilimpregnated film-foil capacitor applications. The films were manufactured either by bubble or tenter film process, however, the exact details on the utilized base polymers and film processing were unknown. Film morphology and surface roughness were characterized by optical profilometry: as is typical for BOPP, one side of the films was smoother than the other, with the rough side exhibiting distinctive crater-like structures (in particular the "hazy" films) [1].

Circular electrodes with diameter of $22 \mathrm{~mm}$ were deposited on both sides of the BOPP film specimens using a custom-built e-beam evaporator (Instrumentti Mattila) inside an ISO 6 class clean room facility. The deposited electrodes comprised of a 10 $\mathrm{nm}$ layer of $\mathrm{Ni}$ (to improve adhesion with BOPP) and a $100 \mathrm{~nm}$ layer of $\mathrm{Au}$. Careful control of the evaporation process and environment is crucial for ensuring that the BOPP film specimen is not damaged during electrode deposition. Especially for semicrystalline materials with relatively low melting temperature such as BOPP, the electrode deposition process can modify the surface trap depth and density, hence affecting the TSDC. High vacuum (pressure $<1 \times 10^{-6} \mathrm{mbar}$ ) and low deposition rate $(0.05-$ $0.20 \mathrm{~nm} / \mathrm{s}$ ) were maintained during the evaporation process to minimize thermal and radiative damaging of the sample. The evaporated samples were short-circuited and stored in desiccator at room temperature. Film thicknesses were measured using a high-resolution thickness gauge.

Table 1. Material specifications.

\begin{tabular}{|c|c|c|c|c|}
\hline \multirow{2}{*}{ Material } & \multirow{2}{*}{ Type } & \multirow{2}{*}{$\begin{array}{c}\text { Thickness } \\
{[\mu \mathrm{m}]}\end{array}$} & \multicolumn{2}{|c|}{ Surface roughness [nm] } \\
\hline & & & Side 1 & Side 2 \\
\hline BOPP-1 & A & $6.6 \pm 0.15$ & $25.0 \pm 4.0$ & $28.3 \pm 2.0$ \\
\hline BOPP-2 & A & $5.1 \pm 0.10$ & $45.6 \pm 22.5$ & $27.2 \pm 8.2$ \\
\hline BOPP-3 & B & $10.0 \pm 0.52$ & $145.0 \pm 13.0$ & $31.0 \pm 3.6$ \\
\hline BOPP-4 & B & $9.1 \pm 0.34$ & $238.6 \pm 24.8$ & $56.8 \pm 9.6$ \\
\hline BOPP-5 & $\mathrm{C}$ & $10.3 \pm 0.39$ & $108.6 \pm 64.4$ & $157.6 \pm 12.2$ \\
\hline
\end{tabular}




\section{TSDC measurement}

The TSDC measurement system consisted of a liquid $\mathrm{N}_{2}-$ based temperature control system with an accuracy of $\pm 0.1{ }^{\circ} \mathrm{C}$ (Novocontrol Novocool), a high voltage DC source (Keithley 2290E-5) and an electrometer (Keithley 6517B). A shielded sample cell equipped with a PT100 temperature sensor (Novocontrol BDS1200 HV sample cell) and shielded cables were used. A diode-based overload protection circuit and a 100 $\mathrm{k} \Omega$ series resistor were utilized to protect the electrometer in case of a sample breakdown during high voltage application (Figure 1a). All TSDC measurements reported in this paper were performed under short-circuit conditions (Figure 1b). The TSDC measurement procedure was as follows:

1) Sample is heated to polarization temperature $T_{p}\left(80-120^{\circ} \mathrm{C}\right)$.

2) Sample is held under isothermal conditions and DC poling field $E_{p}(100-300 \mathrm{~V} / \mu \mathrm{m})$ is applied for time $t_{p}=40 \mathrm{~min}$.

3) Temperature is rapidly decreased to $T_{0}=-50{ }^{\circ} \mathrm{C}$ and maintained for 5 min (voltage on).

4) Voltage is removed; sample is short-circuited and held under isothermal conditions for $3 \mathrm{~min}$.

5) Sample is heated at a linear rate of $\beta\left(3.0-8.0^{\circ} \mathrm{C} / \mathrm{min}\right)$ up to $T_{\max }=\sim 145^{\circ} \mathrm{C}$ while recording the depolarization current.

Making electrical contact with a thin metallized film and subsequently applying high electric field may easily cause a breakdown due to surface imperfections of the contacting electrodes, and this was indeed observed during our initial tests. An improved sample configuration utilized for the TSDC measurements is shown schematically in Figure 1c. A conductive Ag-filled fluorosilicone rubber electrode and a (metal) spring ring was used on the top side of the metallized sample film in order to soften the contact and to distribute the pressure applied to the sample uniformly. The bottom metallized surface of the sample was contacted with a polished Au bulk electrode. This configuration was found to be efficient in preventing mechanical damage and breakdown of the sample upon high voltage application while simultaneously maintaining a good thermal contact to the bottom electrode of the sample holder from which the temperature was measured.

The sample current was measured for the whole duration of the above test cycle. Thus, albeit the polarization time was too short to reach a steady-state DC conduction current, the isothermal charging current data nevertheless provided an indication of the transient electrical conductivity behavior of the sample. It is remarked that the polarization time of $40 \mathrm{~min}$ is too short to determine the "true" DC conductivity of BOPP [2], [3].

\subsection{Additional dielectric measurements}

Complex relative permittivity $\varepsilon_{r}{ }^{*}=\varepsilon_{r}+i \varepsilon_{r}$ ' of the samples was measured using a Novocontrol Alpha-A dielectric analyzer and a Novocool temperature control system under low AC voltage $\left(1 \mathrm{~V}_{\mathrm{rms}}\right)$. The measurements were performed at room temperature conditions as a function of frequency. Additional measurements as a function of frequency and temperature (from $-60{ }^{\circ} \mathrm{C}$ to $130{ }^{\circ} \mathrm{C}$ ) were performed for selected samples. Room temperature DC dielectric breakdown characteristics of the films were measured using a large-area self-healing breakdown approach. The measurements were performed for pristine films
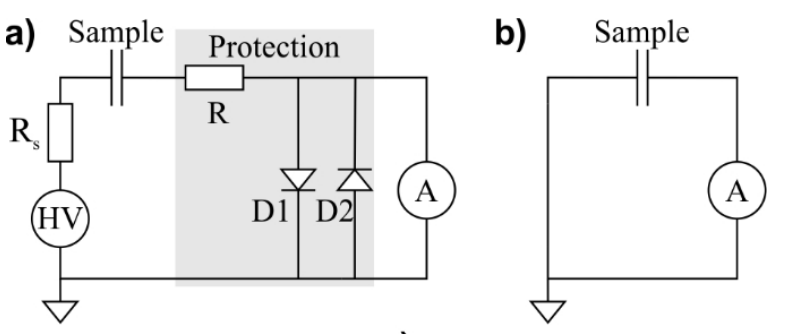

c)

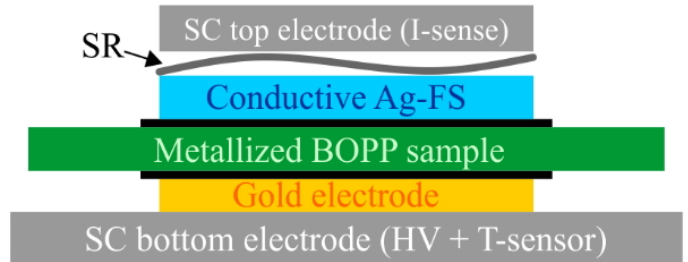

Figure 1. TSDC test circuit during a) polarization and b) depolarization. c) Sample configuration utilized for the TSDC and permittivity measurements. SC: Sample cell, Ag-FS: conductive Agfilled fluorosilicone, SR: spring ring.

with no evaporated electrodes using separate sheets of commercial metallized BOPP film to contact the sample film (active area of $81 \mathrm{~cm}^{2}$ ). Weibull analysis was carried out for the qualified breakdown events. Further details are omitted from this paper and can be found elsewhere [4].

\section{Results and Discussion}

\subsection{TSDC - initial observations}

Figure 2 presents exemplifying TSDC spectra of the studied BOPP films, from which several common observations can be made. Firstly, upon heating from $-50{ }^{\circ} \mathrm{C}$, each film exhibited a broad TSDC peak around $0{ }^{\circ} \mathrm{C}$ which is attributed to the gradual release of charge from shallow traps as the amorphous regions pass through the glass transition ( $\alpha$-relaxation). The $\alpha$-peak temperature range is in good agreement with glass transition temperature determined using differential scanning calorimetry as reported elsewhere [2]. With further heating, strong TSDC peaks were observed in the high temperature region at $T>75^{\circ} \mathrm{C}$, with the peak maximum occurring in the $110-120{ }^{\circ} \mathrm{C}$ range. Given the non-polar nature of BOPP (i.e. lack of dipolar relaxation above glass transition), the high temperature peaks are attributable to the thermal de-trapping of space charge ( $\rho$ relaxation) from deep traps. While TSDC does not provide any direct evidence of the origin of the deep trap states, various complementary methods have indicated that they may be caused by additives (i.e. antioxidants), chemical impurities or melting of less perfect PP crystallites [5]-[7]. Previously, the high temperature TSDC peaks of BOPP have also been associated with ionic conduction [8]. As opposed to the shallow trap $(\alpha-)$ TSDC peaks for which the peak location and intensity was always well repeatable, the TSDC $(\rho-)$ peaks associated with space charge were often less predictable, showing considerable variation in TSDC sign, intensity and location even for parallel samples measured under identical conditions.

Comparing the different film types and manufacturing methods, prominent differences between the studied BOPP films were observed especially in the high temperature $(\rho)$ region. The smooth films manufactured using the tenter process seemed to 


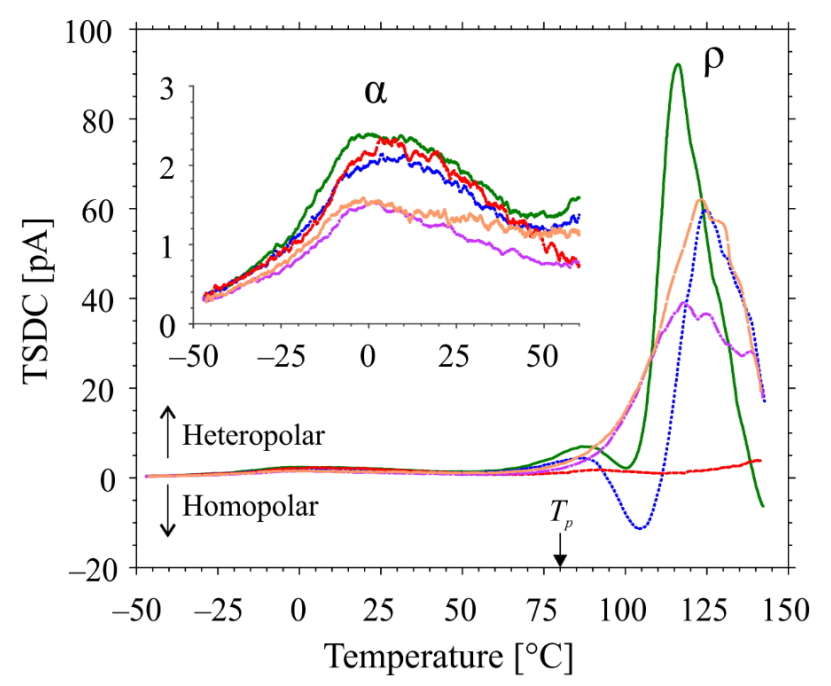

BOPP-1 - BOPP-2 …... BOPP-3 ----- BOPP-4 -.- BOPP-5 --

Figure 2. Exemplifying TSDC spectra of the studied BOPP films $\left(T_{p}=\right.$ $\left.80{ }^{\circ} \mathrm{C}, E_{p}=100 \mathrm{~V} / \mu \mathrm{m}, \beta=3.0^{\circ} \mathrm{C} / \mathrm{min}\right)$. The inset shows a zoomed-in portion of the "shallow trap region" from $-50{ }^{\circ} \mathrm{C}$ to $60{ }^{\circ} \mathrm{C}$.

result in the largest TSDC intensity in the deep trap region. On the other hand, in particular BOPP-3 (a hazy bubble BOPP film) stood out from the rest as it exhibited a significantly lower TSDC intensity in the high temperature (deep trap) region. Such a feature can be indicative of a lower trap density and/or more homogeneous distribution of charge in the specimen [9], as in an ideal case, if the charge distribution was completely uniform in the specimen, the resulting TSDC would be zero [10]. The observed differences can arise from differences in e.g. crystallinity, morphology, film thickness, surface roughness, base PP purity, additives and electrode-polymer interface [9].

\subsection{On the high temperature TSDC polarity reversal}

One of the most intriguing characteristics of the high temperature space charge TSDC peaks is the polarity reversal which was often observed in temperature range $T>T_{p}$. In Figure 2 , the positive current sign indicates a heteropolar current component (TSDC sign opposite to the charging current direction) while the negative current sign indicates a homopolar or "anomalous" [11] current component (TSDC sign same as the charging current). The TSDC polarity reversal is closely related with the polarization conditions upon formation of the electret [11], [12]. With increasing electric field and temperature the injected space charge density and mobility increase, hence leading to increasing amount of homocharge accumulation near the injecting electrode. Moreover, formation of heterocharge layer can occur due to the dissociation of bulk charges (e.g. ionic impurities) or drift of injected homocharge towards the adjacent electrode when the polarization time is sufficiently long. When a specimen with "frozen in" space charge is then short-circuited and subsequently heated, the thermally de-trapped charges drift under the internal field caused by the space charge. As the dynamics of the space charge are then largely dictated by the nature of the dielectric-electrode interface (ohmic vs. partially blocking), carrier diffusion, retrapping propensity etc., the thermally stimulated charge relaxation can evidently become very complex, and a net current flow in the same direction as the charging current can indeed occur [9], [13], [14].

\subsection{TSDC repeatability and the dependence on polarization and depolarization conditions}

In order to investigate the repeatability and the dependence of TSDC on the polarization/depolarization conditions, and to elucidate the nature of the high temperature polarity reversal phenomenon, BOPP-1 was selected for more detailed studies. Several BOPP-1 film specimens were evaporated and subsequently measured using TSDC under various polarization and depolarization conditions. Table 2 summarizes the observations from each experiment, divided into four different cases as will be discussed in the following.

\section{1) Case A: Parallel samples and variable evaporation rate}

Large variation in the high temperature space charge peak temperature, intensity and sign was observed, even for films measured under identical conditions; Table 2, Case A, demonstrates this phenomenon for samples prepared and measured at different occasions. Effects of sample orientation (i.e. which side is facing the HV electrode) and electrode deposition rate were studied, as these aspects can play a role in charge injection process due to the asymmetrical surface/bulk morphology of BOPP films and modification of surface trap structure upon electrode deposition [15]. The space charge peak intensity and polarity reversal were, however, not found to vary systematically when the above factors were changed. The somewhat sporadic nature of the space charge peak is also demonstrated in the following cases.

\section{2) Case B: Variable polarization field}

Table 2, Case B, demonstrates the effect of polarization field on the TSDC. The intensity of the heteropolar shallow trap $(\alpha-)$ peak was found to increase approximately linearly with the poling field while the peak temperature was unaffected. On the other hand, this was not the case for the high temperature $(\rho-)$ peak: the space charge TSDC peak shifted to higher temperature and its intensity increased and saturated with polarization field, with such a non-linear polarization field vs. TSDC intensity being a characteristic feature for space charge [16]. In the $<300$ $\mathrm{V} / \mu \mathrm{m}$ field range, the conductivity of BOPP is known to be more strongly thermally- than field-activated [2]; thus, it is likely that the increasing field in Case $\mathrm{B}$ only resulted in a moderate increase in trap filling during the poling process at $80^{\circ} \mathrm{C}$.

\section{3) Case C: Variable polarization temperature}

Table 2, Case $\mathrm{C}$, demonstrates the effect of polarization temperature on the TSDC. A small increase in heteropolar peak intensity and a slight reduction in peak temperature was observed for the shallow trap $(\alpha-)$ peak. This could be related with morphological changes taking place during isothermal poling at high temperature (thickening of crystallites, relaxation of biaxial orientation etc. [17]). For the high temperature space charge $(\rho-)$ peak, a systematic shift of peak maximum to higher temperatures was observed, with this indicating that high temperature poling favored the filling of deeper traps [18]. Interestingly, the space charge peak intensity decreased significantly when the sample was poled at $120{ }^{\circ} \mathrm{C}$; this is likely a result of the significant increase in charge mobility at $120^{\circ} \mathrm{C}$ as the deep traps became effectively conducting, hence leading to less charge being trapped in the specimen after poling [11]. 
Table 2. Effect of various polarization/depolarization parameters on the TSDC spectra of BOPP-1 divided into different cases. In each figure, the inset shows a zoomed-in portion of the "shallow trap region" from $-50^{\circ} \mathrm{C}$ to $60{ }^{\circ} \mathrm{C}$. The arrows denote the polarization temperature.

Case

Case A:

Parallel samples and variable evaporation rate

$$
\begin{gathered}
E_{p}=100 \mathrm{~V} / \mu \mathrm{m} \\
T_{p}=80^{\circ} \mathrm{C} \\
\beta=3.0^{\circ} \mathrm{C} / \mathrm{min}
\end{gathered}
$$

TSDC spectra

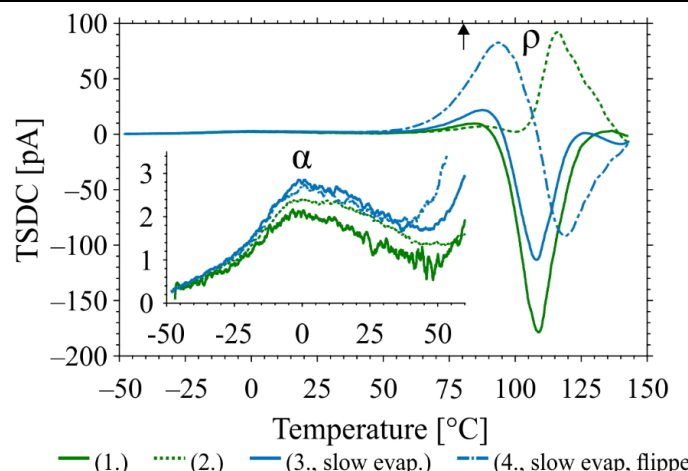

\section{Observations}

Glass-transition region $(\alpha)$ - Shallow traps:

- Heteropolar peak intensity increases slightly when evaporation rate is halved.

- Peak temperature is unaffected.

Space charge peak ( $\rho)$ - Deep traps:

- Polarity reversal may occur above $T_{p}$.

- Intensity of homopolar TSDC component may decrease when evaporation rate is halved.

- No clear effect when sample is flipped.

Glass-transition region $(\alpha)$ - Shallow traps:

- Heteropolar peak intensity increases approximately linearly with poling field.

- Peak temperature is unaffected.

Variable polarization field

$$
\begin{gathered}
T_{p}=80^{\circ} \mathrm{C} \\
\beta=3.0^{\circ} \mathrm{C} / \mathrm{min}
\end{gathered}
$$

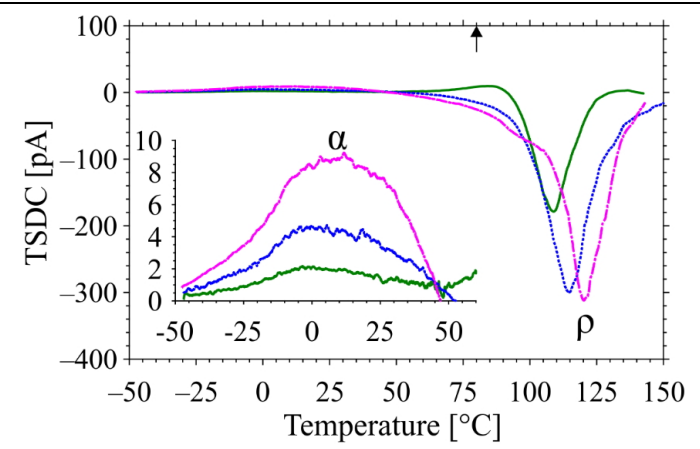

$-E_{p}=100 \mathrm{~V} / \mu \mathrm{m} \quad \cdots \cdot E_{p}=200 \mathrm{~V} / \mu \mathrm{m} \quad--E_{p}=300 \mathrm{~V} / \mu \mathrm{m}$

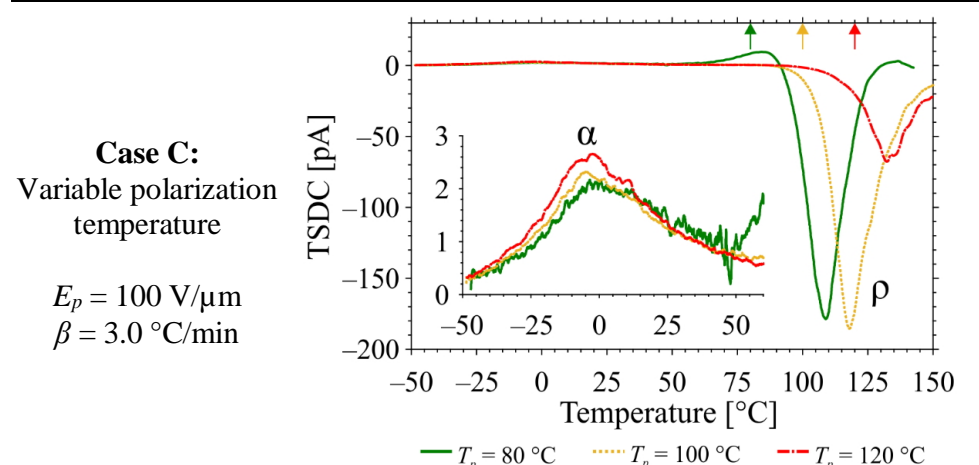

Case D:

Variable heating rate

$$
\begin{gathered}
E_{p}=100 \mathrm{~V} / \mu \mathrm{m} \\
T_{p}=80^{\circ} \mathrm{C}
\end{gathered}
$$

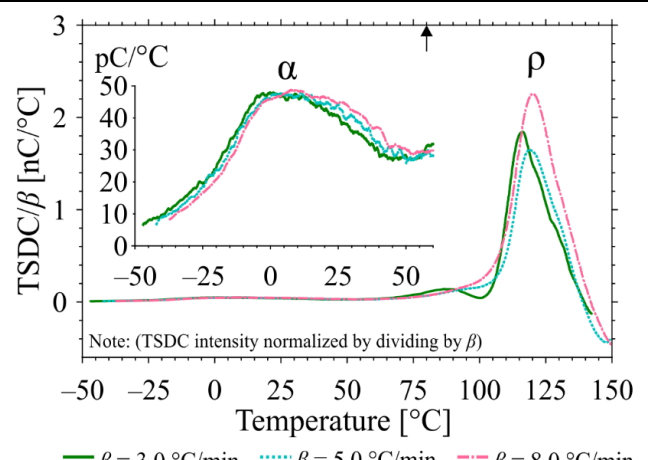

$-\beta=3.0^{\circ} \mathrm{C} / \mathrm{min} \quad \cdots \cdots=5.0^{\circ} \mathrm{C} / \mathrm{min} \quad--\beta=8.0^{\circ} \mathrm{C} / \mathrm{min}$
Space charge peak ( $\rho)$ - Deep traps:

- Polarity reversal may occur above $T_{p}$.

- Homopolar TSDC peak shifts to higher temperature with increasing polarization field $\left(108.8^{\circ} \mathrm{C} \rightarrow 114.7^{\circ} \mathrm{C} \rightarrow 120.3\right.$ $\left.{ }^{\circ} \mathrm{C}\right)$.

- Homopolar TSDC peak intensity increases non-linearly with increasing $E_{p}$.
Glass-transition region $(\alpha)$ - Shallow traps:

- Subtle increase in heteropolar peak intensity.

- Peak temperature is slightly reduced.

Space charge peak $(\rho)$ - Deep traps:

- Polarity reversal may occur above $T_{p}$.

- Homopolar TSDC peak shifts to higher temperature with increasing polarization temperature $\left(108.8^{\circ} \mathrm{C} \rightarrow 118.0^{\circ} \mathrm{C} \rightarrow\right.$ $\left.132.5^{\circ} \mathrm{C}\right)$.

- Homopolar TSDC peak intensity decreases when $T_{p}=120^{\circ} \mathrm{C}$ (increased charge mobility during polarization $\rightarrow$ reduced trapping ability).
Glass-transition region $(\alpha)$ - Shallow traps:

- Normalized heteropolar peak intensity is unaffected.

- Subtle shift of peak temperature to higher temperature with increasing heating rate.

Space charge peak $(\rho)$ - Deep traps:

- Polarity reversal may occur above $T_{p}$.

- Heteropolar peak shifts slightly to higher temperature with increasing heating rate $\left(115.9^{\circ} \mathrm{C} \rightarrow 118.9^{\circ} \mathrm{C} \rightarrow 120.2^{\circ} \mathrm{C}\right)$.

- Normalized TSDC peak intensity and area remains relatively unaffected.

\section{4) Case D: Variable heating rate}

The effect of variable heating rate on the TSDC profile is presented in Table 2, Case D. Comparing the TSDC peaks in normalized units (i.e. by dividing the TSDC by the heating rate $\beta$ ), the area under the curves remained relatively constant [9]. In principle, increasing the heating rate should increase the temperature of the TSDC peak maximum, and this was indeed generally observed. The peak shift with varying heating rate provides a way to determine the associated thermal activation energy [19]. It is however important to note that slow morphological changes can take place during the high temperature polarization/depolarization phases, and this may have an impact on the accuracy of the determination of activation energy from variable-heating-rate TSDC data. 


\subsection{On the estimation of trap parameters from TSDC}

Polymers often exhibit complex TSDC spectra due to several overlapping or (quasi-) continuous trap bands, with this making the estimation of the trapping parameters from TSDC data far from trivial [9]. Indeed, application of simple methods such as the initial-rise method or peak shape methods, which assume single isolated TSDC peaks, is often unfeasible, and more intricate methods involving curve-fitting are needed [16]. Under slow re-trapping conditions (i.e. $1^{\text {st }}$ order kinetics), the thermally stimulated current arising from a single discrete trap depth under linear heating is given by the Randall-Wilkins equation:

$$
I(T)=I_{0} \exp \left(-\frac{E}{k T}\right) \exp \left(-\frac{v}{\beta} \int_{T_{0}}^{T} \exp \left(-\frac{E}{k T}\right) d T\right)
$$

where $T$ is temperature, $E$ is the trap depth, $k$ is the Boltzmann constant, $\beta$ is the linear heating rate, $T_{0}$ is the initial temperature before heating and $I_{0}$ is a constant and $v$ is the attempt-to-escape frequency. In principle, a complex TSDC curve can be decomposed into $n$ elementary peaks, with each peak given by Equation (1), using e.g. non-linear curve fitting in MATLAB. As proposed by Neagu et al., a complex TSDC peak can be decomposed step-by-step by first fitting the most prominent peak, subtracting it from the data and then continuing this procedure for the remaining side peaks until a satisfactory fit is achieved [16]. For the BOPP films studied here, the polarity reversal phenomenon in the high temperature region poses a further challenge for curve fitting [20]. Multi-peak fitting is exemplified in Figure 3 where the complex TSDC curve of a BOPP-1 specimen was decomposed into five elementary peaks

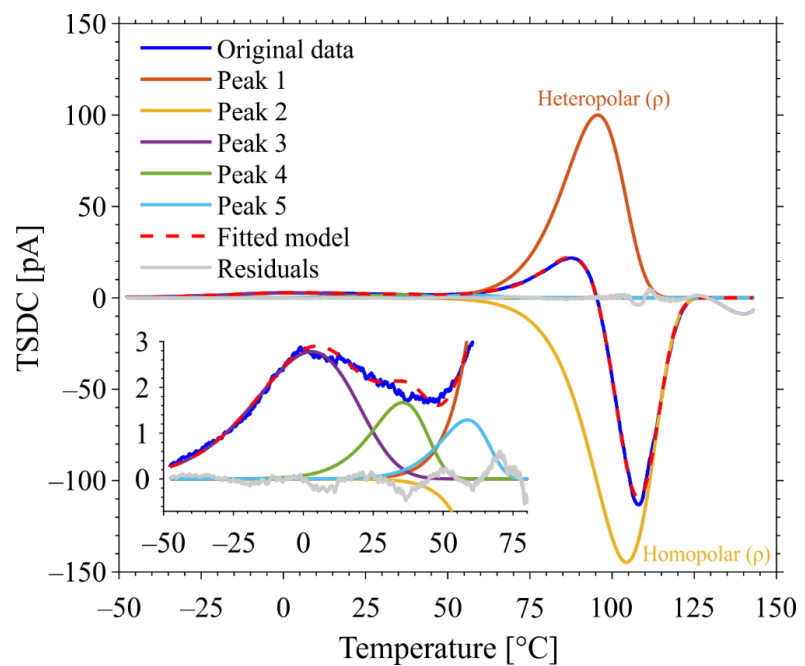

Figure 3. Example of multi-peak fitting applied to TSDC data from a BOPP-1 specimen. The inset shows a close-up of the shallow trap region (from -50 to $80{ }^{\circ} \mathrm{C}$ ). Peak parameters are given in Table 3 .

Table 3. Trap parameters of the five peaks from Figure 3. $T_{m}$ and $I_{m}$ are the temperature and current at peak maximum. $Q_{0}$ is the total charge.

\begin{tabular}{crrrrr}
\hline Peak & $\boldsymbol{T}_{\boldsymbol{m}}\left[{ }^{\circ} \mathbf{C}\right]$ & $\boldsymbol{I}_{\boldsymbol{m}}[\mathbf{p A}]$ & $\boldsymbol{E}[\mathbf{e V}]$ & $\boldsymbol{Q}_{\boldsymbol{0}}[\mathbf{n C}]$ & $\boldsymbol{v}[\mathbf{1} / \mathbf{s}]$ \\
\hline 1 & 2.6 & 2.8 & 0.34 & 2.54 & $4.00 \times 10^{3}$ \\
2 & 35.5 & 1.7 & 0.82 & 0.85 & $1.41 \times 10^{11}$ \\
3 & 58.7 & 1.3 & 1.08 & 0.58 & $1.53 \times 10^{14}$ \\
4 & 95.6 & 100.2 & 1.27 & 47.88 & $1.15 \times 10^{15}$ \\
5 & 104.4 & -144.7 & 1.30 & -70.83 & $1.11 \times 10^{15}$ \\
\hline
\end{tabular}

using the above procedure and taking into account the presence of both homo- and heteropolar components in the space charge region [20]. Although a good fit does not necessarily mean correct deconvolution, the trap parameters given in Table 3 are nevertheless within physically acceptable range for a thermally stimulated process.

The above case example only considers the slow retrapping case; other TSDC models taking into account the charge retrapping process such as the general order kinetics model [21] were also studied. For the other methods, we also applied a recently popular numerical method [22], originally proposed by Simmons [23], providing a way to approximate the continuous density of states distribution directly from the TSDC data. The model, however, only considers the slow electron retrapping case and several parameters such as the attempt-to-escape frequency and charge penetration depth need to be assumed. Alternatively, the "Direct Signal Analysis" method proposed by Aldana et al. [24] can provide a more thorough way for the evaluation of continuous trap spectra: the method involves dividing the energy range of interest into uniformly distributed energy bins and reconstructing the trap spectra using elementary peak equations (however, assumptions on the trap energy range and bin width still need to be done). For the sake of brevity these results are not discussed further here. It is anyhow clear that the accurate derivation of trap parameters from TSDC data is not straightforward and several assumptions on the underlying trapping/de-trapping/re-trapping phenomena have to be made. Utilization of complementary methods for derivation of trap parameters in conjunction with TSDC is thus seen necessary, as has been done elsewhere [25]-[27].

\subsection{Relation of TSDC with other dielectric properties}

Dielectric spectroscopy, high-field conductivity and room temperature DC breakdown characteristics of the studied BOPP films are summarized in Table 4. All the films exhibited very low dielectric loss characteristics (tan $\delta \sim 10^{-4}$ ), low conductivity (in the $10^{-16}$ to $10^{-15} \mathrm{~S} / \mathrm{m}$ range) and high dielectric strength (Weibull $\alpha$ of $680-770 \mathrm{~V} / \mu \mathrm{m}$ ) which is typical for capacitor-grade BOPP. Good correlation with the corresponding TSDC data was observed for temperature-dependent dielectric loss data (Figure 4) and high-field conductivity data (Table 4): the temperature-dependent $\tan \delta$ showed similar $\alpha$ - and $\rho$ relaxations as the corresponding TSDC spectra, while higher conductivity (at the end of the isothermal polarization period) was seemingly correlated with a higher TSDC deep trap intensity close to $T_{p}$ (see Figure 2). On the other hand, linking the room temperature DC ramp breakdown strength with the TSDC data may be less straightforward, as morphological factors such as crystalline structure and surface roughness may

Table 4. Permittivity (at $1 \mathrm{kHz}$ ), mean conductivity (at $80{ }^{\circ} \mathrm{C}, 100$ $\mathrm{V} / \mu \mathrm{m}$; after $40 \mathrm{~min}$ poling) and DC dielectric strength results. $n$ is the exponent of the power law decay of current $I$ over time $t\left(I \sim t^{-n}\right) . \alpha$ and $\beta$ are the Weibull shape and scale parameters, respectively.

\begin{tabular}{|c|c|c|c|c|c|c|}
\hline \multirow{2}{*}{ Material } & \multicolumn{2}{|c|}{ Permittivity } & \multicolumn{2}{|c|}{ Conductivity } & \multicolumn{2}{|c|}{ DBS } \\
\hline & $\varepsilon_{r}$ & $\tan \delta$ & $\sigma(\mathrm{S} / \mathrm{m})$ & $n$ & $\alpha(\mathrm{V} / \mu \mathrm{m})$ & $\beta$ \\
\hline BOPP-1 & 2.15 & $1.61 \times 10^{-4}$ & $3.21 \times 10^{-15}$ & 0.73 & 771 & 19.2 \\
\hline BOPP-2 & 2.15 & $1.60 \times 10^{-4}$ & $2.46 \times 10^{-15}$ & 0.71 & 704 & 23.3 \\
\hline BOPP-3 & 2.17 & $1.66 \times 10^{-4}$ & $9.28 \times 10^{-16}$ & 0.64 & 713 & 16.2 \\
\hline BOPP-4 & 2.16 & $1.08 \times 10^{-4}$ & $1.86 \times 10^{-15}$ & 0.66 & 686 & 23.8 \\
\hline BOPP-5 & 2.19 & $1.12 \times 10^{-4}$ & $1.55 \times 10^{-15}$ & 0.62 & 681 & 29.5 \\
\hline
\end{tabular}




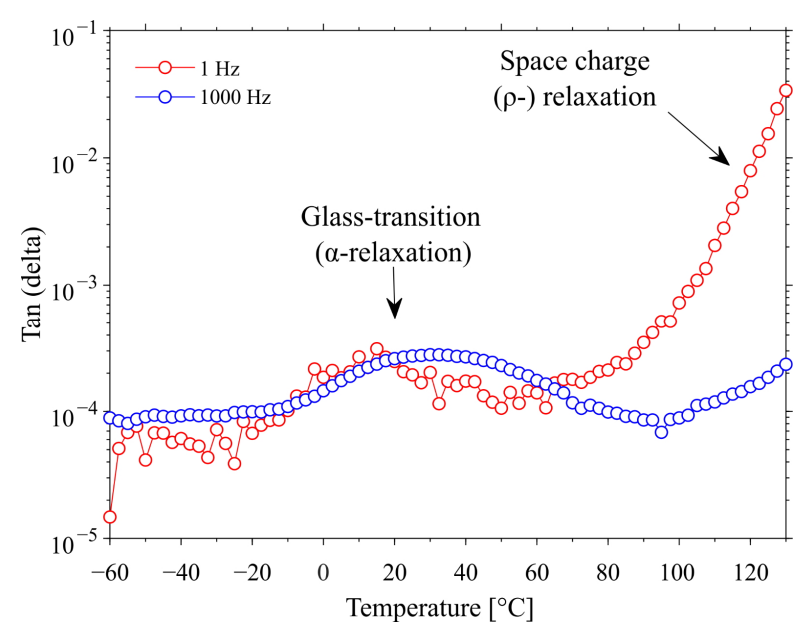

Figure 4. Temperature-dependent dielectric loss ( $\tan \delta)$ of BOPP-1 at $1 \mathrm{~Hz}$ and $1 \mathrm{kHz}$ (sample measured after TSDC).

be more determining factors for DC breakdown strength under short-term conditions [1] rather than homocharge formation near the electrodes (i.e. suppression of charge injection and enhancement of bulk field) [28]. Nevertheless, the measurement of high-temperature breakdown strength and/or voltage endurance [29], and their relationship with the deep trap TSDC spectra, are of interest, and such work will be carried out in a future study.

\section{Conclusions}

Aspects on the measurement and interpretation of thermally stimulated currents of various capacitor-grade BOPP films have been investigated, demonstrating both the usefulness and the challenges of the TSDC method in elucidating the trapping characteristics of capacitor films. The main TSDC peaks observed in the $-50{ }^{\circ} \mathrm{C}$ to $145{ }^{\circ} \mathrm{C}$ temperature range are associated with the glass transition ( $\alpha$-relaxation) and space charge relaxation ( $\rho$-relaxation), with both of these giving rise to complex discharge current spectra which cannot be described by single discrete trap models alone. The high temperature TSDC polarity reversal phenomenon associated with the injected space charge further complicates the TSDC analysis, and consideration of both homo- and heteropolar components during decomposition of TSDC spectra is necessary. Despite the complications however, the results indicate that the observed differences between TSDC trap spectra of various tenter and bubble BOPP films can be related with their morphology and other dielectric properties.

\section{References}

[1] I. Rytöluoto, A. Gitsas, S. Pasanen, and K. Lahti, "Effect of film structure and morphology on the dielectric breakdown characteristics of cast and biaxially oriented polypropylene films," Eur. Polym. J., vol. 95, 2017.

[2] I. Rytöluoto, M. Ritamäki, and K. Lahti, "Short-term dielectric performance assessment of BOPP capacitor films: A baseline study," in 12th Int'l Conf. Prop. App. Dielec. Mat. (ICPADM), 2018, pp. 289-292.

[3] H. Ghorbani et al., "Long-term conductivity decrease of polyethylene and polypropylene insulation materials," IEEE Trans. Dielectr. Electr. Insul., vol. 24, no. 3, pp. 1485-1493, Jun. 2017.

[4] I. Rytöluoto, K. Lahti, M. Karttunen, and M. Koponen, "Large-area dielectric breakdown performance of polymer films - Part I: Measurement method evaluation and statistical considerations on area-dependence," IEEE Trans. Dielectr. Electr. Insul., vol. 22, no. 2, pp. 689-700, 2015.

[5] H.-V. Nguyen and T. H. Pham, "Structural and Electronic Properties of
Defect-Free and Defect-Containing Polypropylene: A Computational Study by van der Waals Density-Functional Method," Phys. Status Solidi, p. 1700036 , Oct. 2017.

[6] T. Umemura, T. Suzuki, and T. Kashiwazaki, "Impurity Effect of the Dielectric Properties of Isotactic Polypropylene," IEEE Trans. Electr. Insul., vol. EI-17, no. 4, pp. 300-305, 1982.

[7] P. Myśliński and M. Kryszewski, "The effect of crystalline structure on the thermally stimulated discharge current of isotactic polypropylene," Acta Polymerica, vol. 38, no. 5, pp. 253-258, 1987.

[8] A. Kahouli, O. Gallot-Lavallée, P. Rain, O. Lesaint, C. Guillermin, and J.M. Lupin, "Dielectric features of two grades of bi-oriented isotactic polypropylene," J. Appl. Polym. Sci., vol. 132, no. 28, p. 42224, 2015.

[9] G. M. Sessler, Ed., Electrets, vol. 33. Berlin, Heidelberg: Springer, 1987.

[10] K.-S. Suh, J. Tanaka, and D. Damon, "What is TSC?," IEEE Electr. Insul. Mag., vol. 8, no. 6, pp. 13-20, Nov. 1992.

[11] P. K. Khare and S. K. Jain, "Anomalous thermally stimulated currents and space charge in poly(vinyl pyrrolidone)," Polym. Int., vol. 49, no. 3, pp. 265-268, Mar. 2000.

[12] T. Hashimoto, M. Shiraki, and T. Sakai, "Current reversal in the thermally stimulated current spectra of polyethylene," J. Polym. Sci. Polym. Phys. Ed., vol. 13, no. 12, pp. 2401-2410, Dec. 1975.

[13] H. J. Wintle, "Reversals in Electrical Current and Other Anomalies in Insulating Polymers," IEEE Trans. Electr. Insul., vol. EI-21, no. 5, pp. 747-762, 1986.

[14] B. Gross and M. M. Perlman, "Short-circuit currents in charged dielectrics and motion of zero-field planes," J. Appl. Phys., vol. 43, no. 3, pp. 853$855,1972$.

[15] A. Thielen, J. Niezette, G. Feyder, and J. Vanderschueren, “Thermally stimulated current study of space charge formation and contact effects in metal-polyethylene terephthalate film-metal systems. I. Generalities and theoretical model," J. Phys. Chem. Solids, vol. 57, no. 11, pp. 1567-1580, 1996.

[16] R. M. Neagu, E. R. Neagu, I. M. Kalogeras, and A. Vassilikou-Dova, "Evaluation of the dielectric parameters from TSDC spectra: Application to polymeric systems," Mater. Res. Innov., vol. 4, no. 2-3, pp. 115-125, 2001.

[17] F. Zheng, M. Gu, J. Dong, Z. An, Q. Lei, and Y. Zhang, "Fast space charge behavior in heat-treated polypropylene films," J. Appl. Polym. Sci., vol. 132, no. 28, 2015.

[18] S. C. Datt, R. S. Baghel, and R. Singh, "Normal and anomalous thermally stimulated currents and space charge in polypropylene," in Proc. 6th Int'l Symp. Electrets (ISE 6), 1988, pp. 246-250.

[19] R. Chen and S. A. A. Winer, "Effects of Various Heating Rates on Glow Curves," J. Appl. Phys., vol. 41, no. 13, pp. 5227-5232, Dec. 1970.

[20] J. Belana, J. A. Diego, J. Orrit, I. Tamayo, and M. Mudarra, "Study of an initial transient relaxation in XLPE cable insulation by TSDC and PEA," IEEE Trans. Dielectr. Electr. Insul., vol. 18, no. 6, pp. 2074-2082, 2011.

[21] M. Mudarra and J. Belana, "Study of poly(methyl methacrylate) space charge relaxation by TSDC," Polymer, vol. 38, no. 23, pp. 5815-5821, 1997.

[22] F. Tian, W. Bu, L. Shi, C. Yang, Y. Wang, and Q. Lei, "Theory of modified thermally stimulated current and direct determination of trap level distribution," J. Electrostat., vol. 69, no. 1, pp. 7-10, Feb. 2011.

[23] J. G. Simmons, G. W. Taylor, and M. C. Tam, "Thermally Stimulated Currents in Semiconductors and Insulators Having Arbitrary Trap Distributions," Phys. Rev. B, vol. 7, no. 8, pp. 3714-3719, Apr. 1973.

[24] M. Aldana, E. Laredo, A. Bello, and N. Suarez, "Direct signal analysis applied to the determination of the relaxation parameters from TSDC spectra of polymers," J. Polym. Sci. Part B Polym. Phys., vol. 32, no. 13, pp. 2197-2206, Oct. 1994.

[25] I. Rytöluoto et al., "Compounding, Structure and Dielectric Properties of Silica-BOPP Nanocomposite Films," in 2nd Int'l Conf. Dielec., 2018.

[26] M. Carmo Lanca et al., "Comparative study of space charge in the polymeric insulation of power cables using PEA, isothermal and nonisothermal currents measurements," in 12th Int'l Symp. Electrets, 2005, pp. 284-287.

[27] L. Boudou, F. Zheng, and G. Teyssedre, "Photo-stimulated discharge current measurements on biaxially oriented polypropylene thin films (BOPP)," Proc. IEEE Int. Conf. Prop. Appl. Dielectr. Mater., vol. 2018May, pp. 722-725, 2018.

[28] L. Cheng, W. Liu, X. Liu, C. Liu, S. Li, and Z. Xing, "Online degradation of biaxial-orientated polypropylene film from HVDC filter capacitors," IEEE Trans. Dielectr. Electr. Insul., vol. 26, no. 1, pp. 26-33, Feb. 2019.

[29] M. Ritamäki, I. Rytöluoto, and K. Lahti, "DC Voltage Endurance of Capacitor BOPP Films at High Temperature," in Int'l Conf. Dielec., 2018. 